\title{
Symmetric rate model for fluorocarbon plasma etching of $\mathrm{SiO}_{2}$
}

\author{
Ji Ding ${ }^{\text {a) }}$ and Noah Hershkowitz \\ Engineering Research Center for Plasma-Aided Manufacturing, University of Wisconsin-Madison, Madison, \\ Wisconsin 53706
}

(Received 27 February 1995; accepted for publication 16 January 1996)

\begin{abstract}
A symmetric rate model for plasma etching and plasma deposition in fluorocarbon plasmas is proposed. When there is no deposition, the symmetric rate model gives a plasma etch rate. When there is no etching, the model gives a plasma deposition rate. Electron cyclotron resonance and reactive ion etcher etch rates of $\mathrm{SiO}_{2}$ in $\mathrm{CF}_{4}$ plasma are found to be consistent with the model.

(C) 1996 American Institute of Physics. [S0003-6951(96)02212-3]
\end{abstract}

Plasma etching is an important technology in the fabrication of very large scale integrated circuits. ${ }^{1}$ With the feature size smaller than $0.5 \mu \mathrm{m}$, the empirical approach for the optimization of plasma etching is getting more and more difficult, and basic understanding of plasma etching is becoming crucial for process control. In the etching of $\mathrm{SiO}_{2}$ with fluorocarbon plasmas, both etching and deposition occur simultaneously ${ }^{2}$ and both processes need to be understood.

Many rate models have been proposed for plasma etching (see Refs. 3-8 and references therein). Among these models, Zawaideh and $\mathrm{Kim}^{7}$ proposed a model to include both linear effects of chemical and physical etching, and the nonlinear effect of "enhanced" chemical and physical etching. Hoffmann and Heinrich ${ }^{8}$ simplified the model proposed by Zawaideh and Kim to describe reactive ion etching (RIE) of polysilicon with $\mathrm{SF}_{6}$ plasma. Gottscho, Jurgensen, and Vitkavage ${ }^{10}$ proposed an ion-neutral synergy model based on the mass transport model proposed by Mayer and Barker. ${ }^{3,5}$ In this letter, a symmetric rate model for plasma etching and deposition is developed.

In both plasma etching and deposition, energetic ions, etching species, and deposition species have been shown to be important. ${ }^{9-14}$ Fracassi et al..$^{9}$ showed that plasma etching and deposition rates of plasma perfluoropolymer thin films have a similar dependence on ion energy. Ding et al. ${ }^{10}$ found in an electron cyclotron resonance (ECR) etcher that the etch rates of $\mathrm{SiO}_{2}$ with $\mathrm{CF}_{4} / \mathrm{O}_{2} / \mathrm{Ar}$ plasmas only depend on ion energy flux and $\mathrm{F}$-atom density. They quantified the boundary between the ion energy flux limited regime and the F-atom flux limited regime. Mutsukura et al. ${ }^{11}$ studied the deposition of hydrogenated hard-carbon films in a $\mathrm{CH}_{4} \mathrm{rf}$ discharge plasma. They found at high pressure that the film deposition rate was predominantly dependent on the ion energy flux. They also found at very low pressure that the film deposition rates were almost the same for different rf power at each pressure condition. Similar trends have also been found by the other groups. ${ }^{12-14}$

Based on the experimental results and the models mentioned earlier, we assume that the ion enhanced chemical etch rate and the ion enhanced chemical deposition rate are proportional to the ion energy flux multiplied by the surface coverage of the etching or deposition species. This assump-

${ }^{a}$ Current address: Applied Materials, Santa Clara, CA. tion means the etch rate and deposition rate are symmetric. If the products of the reaction are volatile, then the process results in net etching. If the products of the reaction are involatile, then the process results in net deposition. The energy deposited onto the surface accelerates both etching and deposition. This symmetric effect has been shown in the work of Fracassi, Occhiello, and Coburn. ${ }^{9}$

In the fluorocarbon plasma etching of $\mathrm{SiO}_{2}$, both plasma etching and deposition (or polymerization) occur. For steady state, there are four main processes: (1) etching of the substrate; (2) deposition (or polymerization) on the substrate; (3) etching of the polymer; (4) deposition on the polymer. The polymer is a fluorocarbon thin film deposited on the substrate. From the assumption described earlier, we write the following equations

$$
\begin{aligned}
& (E R)_{i \alpha}=K_{e \alpha} \Theta_{e \alpha} J_{i} E_{i}, \\
& (D R)_{i \alpha}=K_{d \alpha} \Theta_{d \alpha} J_{i} E_{i},
\end{aligned}
$$

where $\alpha=s, p$ corresponds to substrate or polymer. $(E R)_{i \alpha}$ and $(D R)_{i \alpha}$ are the ion enhanced chemical etching rate and the deposition rate on the substrate or polymer, $K_{e \alpha}$ and $K_{d \alpha}$ are the volumes removed (or grown) per unit bombardment energy $\left(\mathrm{cm}^{3} / \mathrm{eV}\right)$ on the substrate and on the polymer (deposited film), $\Theta_{e \alpha}$ and $\Theta_{d \alpha}$ are the surface coverage of the etching (or deposition) species on the substrate and on the polymer, $J_{i}$ is the ion flux $\left(\mathrm{cm}^{-2} \mathrm{~s}^{-1}\right)$ to the surface, $E_{i}$ the average ion energy in $\mathrm{eV}$, and $J_{i} E_{i}$ is the ion energy flux $\left(\mathrm{eV} / \mathrm{cm}^{2} \mathrm{~s}\right)$.

For steady state, the etching species flux removed from the surface and the incoming etching species flux sticking on the surface must balance, and the film growth rate and the incoming deposition species flux sticking on the surface must also balance. The etching species removed from the surface refers to those etching species carried by the etch products. These balances combined with the Eqs. (1) and (2) give:

$\frac{K_{\mathrm{es}}}{V_{\mathrm{es}}} \Theta_{\gamma s} J_{i} E_{i}=S_{\gamma s}\left(1-\Theta_{\mathrm{es}}-\Theta_{\mathrm{ds}}-\Theta_{p}\right) J_{\gamma}$

(etching of or deposition on substrate), 
$\frac{K_{\mathrm{ep}}}{V_{\mathrm{ep}}} \Theta_{\gamma p} J_{i} E_{i}=S_{\gamma p}\left(\Theta_{p}-\Theta_{\mathrm{ep}}-\Theta_{\mathrm{dp}}\right) J_{e}$

(etching of or deposition on polymer),

where $\gamma=e$ or $d, \Theta_{p}$ is the surface coverage of the polymer on the substrate, $V_{e \alpha}$ is the volume $\left(\mathrm{cm}^{3}\right)$ removed per etching species on the substrate (or polymer), $V_{d \alpha}$ is the volumes $\left(\mathrm{cm}^{3}\right)$ grown per deposition (or polymerization) species on the substrate or polymer, $S_{e \alpha}$ is the reactive sticking probabilities (unitless) of etching species on the bare substrate (or bare polymer), $S_{d \alpha}$ is the reactive sticking probabilities (unitless) of deposition species on the bare substrate (or the bare polymer), and $J_{\alpha}$ is the etching (deposition) species flux to the surface.

The left-hand sides in Eqs. (3) and (4) represent the removing etching species flux carried by the etch products, or the growing deposition species flux inside the film (polymer). The right-hand sides in Eqs. (3) and (4), represent the incoming etching species flux or deposition species flux sticking on the bare substrate or on the bare polymer. For steady state, the polymer coverage does not change. This means that the polymer etch rate $(E R)_{\mathrm{ip}}$ will balance the rate of deposition on the substrate $(D R)_{\text {is }}$. After taking into account the four types of reactions on the substrate given by Eqs. (1) and (2), we find the total time average surface reaction rate is

$$
\begin{aligned}
R_{t} & =(E R)_{\mathrm{is}}-(D R)_{\mathrm{is}}+(E R)_{\mathrm{ip}}-(D R)_{\mathrm{ip}} \\
& =(E R)_{\mathrm{is}}-(D R)_{\mathrm{ip}} .
\end{aligned}
$$

Solving Eqs. (1)-(5), gives the total time average reaction rate:

$$
R_{t}=\frac{K_{\mathrm{es}} J_{i} E_{i}\left[1-C_{\mathrm{sp}}\left(\frac{J_{d}}{J_{e}}\right)^{2}\right]}{1+A_{s} \frac{J_{i} E_{i}}{J_{e}}+B_{s} \frac{J_{d}}{J_{e}}\left[1+\frac{K_{\mathrm{ds}}}{K_{\mathrm{ep}}}\left(1+A_{p} \frac{J_{i} E_{i}}{J_{e}}+B_{p} \frac{J_{d}}{J_{e}}\right)\right]},
$$

where

$$
\begin{aligned}
& A_{\alpha}=\frac{K_{e \alpha}}{V_{e \alpha} S_{e \alpha}}, \quad B_{\alpha}=\frac{K_{e \alpha} V_{d \alpha} S_{d \alpha}}{K_{d \alpha} V_{e \alpha} S_{e \alpha}} \text { and } \\
& C_{\mathrm{sp}}=B_{s} B_{p} \frac{K_{\mathrm{ds}} K_{\mathrm{dp}}}{K_{\mathrm{es}} K_{\mathrm{ep}}}=\frac{V_{\mathrm{ds}} S_{\mathrm{ds}}}{V_{\mathrm{es}} S_{\mathrm{es}}} \frac{V_{\mathrm{dp}} S_{\mathrm{dp}}}{V_{\mathrm{ep}} S_{\mathrm{dp}}}
\end{aligned}
$$

If there is no deposition, $J_{d}=0$ and the total reaction rate given by Eq. (6) reduces to the plasma etch rate given by Gottscho, Jurgensen, and Vitkavage ${ }^{6}$

$$
R_{t}=\frac{K_{\mathrm{es}} J_{i} E_{i}}{1+\frac{K_{\mathrm{es}}}{V_{\mathrm{es}} S_{\mathrm{es}}} \frac{J_{i} E_{i}}{J_{e}}} .
$$

If there is no etching, $J_{e}=0, R_{t}$ gives a plasma deposition rate (indicated by - ),

$$
R_{t}=-\frac{K_{\mathrm{dp}} J_{i} E_{i}}{1+\frac{K_{\mathrm{dp}}}{V_{\mathrm{dp}} S_{\mathrm{dp}}} \frac{J_{i} E_{i}}{J_{d}}} .
$$

The symmetry between etching and deposition given by Eq. (6) is very clear.

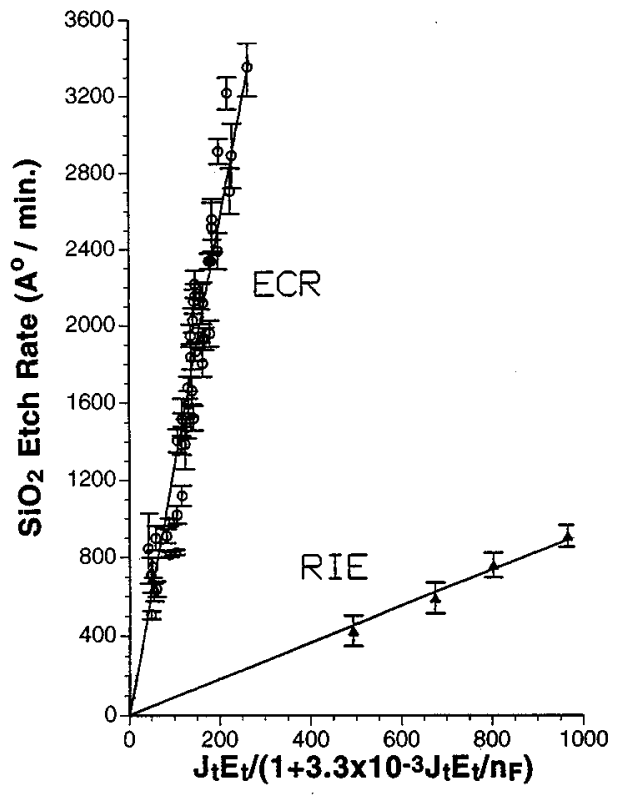

FIG. 1. ECR etch rate data (500-900 W, 0.5-2.5 mTorr, self-bias voltage -25 to $100 \mathrm{~V})$ and RIE data $(700-1500 \mathrm{~W}, 37 \mathrm{mTorr})$ fit to Eq. (8). $J_{i} E_{i}$ is in $\mathrm{mW} / \mathrm{cm}^{2}$ and $n_{F}$ is in $10^{12} \mathrm{~cm}^{-3}$.

Experiments were carried out with an ECR etcher based on an ASTeX (Model S1000) $2.45 \mathrm{GHz}$ source operated at $500-900 \mathrm{~W}, 0.5-2.5$ mTorr, self-bias voltage $=-25$ to 100 $\mathrm{V}$, and flow rates $4-44 \mathrm{sccm}$. rf at $13.56 \mathrm{MHz}$ was used to self-bias the wafer. The wafer was mechanically clamped to a water-cooled wafer stage. RIE experiments were carried out with a Plasma Therm parallel plate etcher (Model 2484) operated with $700-1500 \mathrm{~W}$ of rf at $13.56 \mathrm{MHz}, 37 \mathrm{mTorr}$, and flow rate $=20 \mathrm{sccm}$. Ion flux $J_{i}$ was measured with Langmuir probes, plasma potential with emissive probes and Langmuir probes, fluorine concentration by Ar actinometry, ${ }^{15}$ and $\mathrm{CF}_{2}$ concentration using diode laser infrared absorption. ${ }^{16}$

Figure 1 gives a comparison of the measured $\mathrm{SiO}_{2}$ etch rate by a $\mathrm{CF}_{4}$ plasma, in the $\mathrm{ECR}^{10}$ and RIE tools, with the etch rate given by Eq. (8), the case of no deposition. In Fig. 1 , the ion energy flux $J_{i} E_{i}$ is in $\mathrm{mW} / \mathrm{cm}^{2}$, the F-atom density $n_{\mathrm{F}}$ is in $10^{12} \mathrm{~cm}^{-3}$, and the F-atom temperature is taken as a constant. The etch rates in both ECR and RIE tools have a similar trend and can be fit by Eq. (8). However, the etch rates in the RIE are lower than those in the ECR. For the ECR tool $K_{\text {es }}=13$ while for the RIE tool $K_{\text {es }}=0.93$. For $\mathrm{CF}_{4}$ etching, the deposition species appears to be $\mathrm{CF}_{2}$.

We can simplify Eq. (8) for RIE etching and deposition by assuming $J_{d} / J_{i} E_{i} \ll A_{\alpha} / B_{\alpha}$

$$
R_{t}=\frac{K_{\mathrm{es}} J_{i} E_{i}\left[1-C_{\mathrm{sp}}\left(\frac{J_{d}}{J_{e}}\right)^{2}\right]}{1+A_{s} \frac{J_{i} E_{i}}{J_{e}}} .
$$

It has been proposed that the $\left(\mathrm{CF}_{2}\right)_{n}$ species are the precursors to plasma polymerization of fluorocarbon of $\mathrm{F} / \mathrm{C} \geqslant 2$, since the gas concentration of these species is found to be directly related to deposition rate for a variety of species. ${ }^{2}$ 


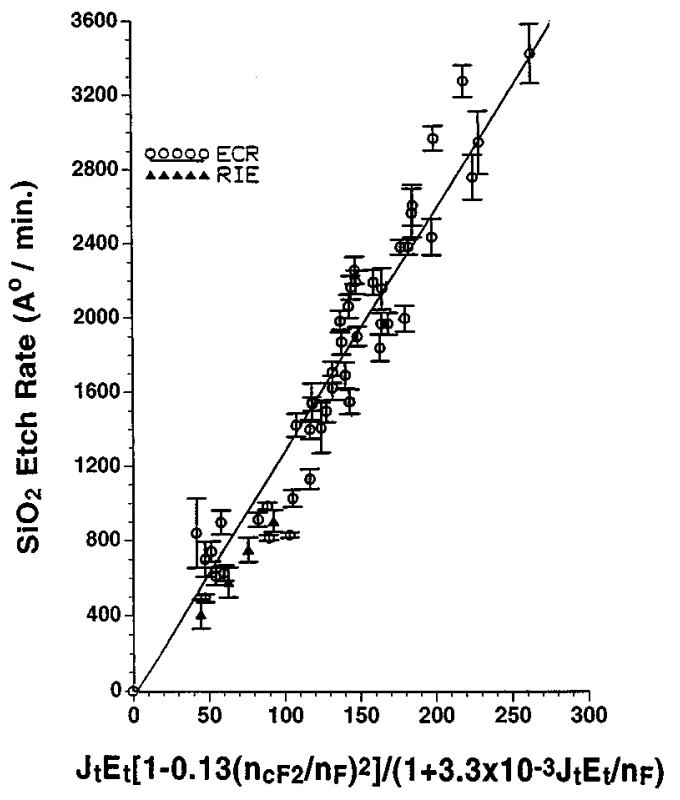

FIG. 2. Etch rate data from the ECR and RIE tools [given in Fig. 1, fit to Eq. (10)].

Therefore, we assume that $\mathrm{CF}_{2}$ is the polymerization species to a first order approximation. Figure 2 gives a comparison of the etch rate in both ECR and RIE with the etch rate given by Eq. (10). Here, the temperatures of $\mathrm{F}$ atoms and $\mathrm{CF}_{2}$ radicals have been assumed to be the same. In fitting Eq. (10) to the data, all constants have been treated as free parameters. Fit values of the constants $C_{s p}$ and $A_{s}$ are shown on Fig. 2. The constant $K_{e s}$ is found to be 13. Our measurements find that the ratio $\left(J_{d} / J_{e}\right)$ is small (the order of $10^{-2}$ ) for $\mathrm{CF}_{4}$ etching of $\mathrm{SiO}_{2}$ in the ECR tool but that it is significant $(\approx 2.7)$ in the RIE tool. The difference in ECR and RIE etch rates can be understood as the result of polymer deposition on the wafer.
In summary, a symmetric rate model has been developed for plasma etching and deposition. In the case of no deposition, the symmetric rate model reduces to the plasma etching rate given by Gottscho, Jurgensen, and Vitkavage. ${ }^{6}$ In the case of no etching, the symmetric rate model gives a plasma deposition rate symmetric to the plasma etching rate. All ECR etching results are explained by the symmetric rate model in the case of no deposition. The RIE results are modified by a factor of $\left[1-C_{s p}\left(J_{d} / J_{e}\right)^{2}\right]$, a simplified case of the symmetric rate model. In this case, the etch rates in the ECR and RIE tools are derived by an equation which depends on only three constants.

This work was supported by NSF Grant EEC 8721545 .

${ }^{1}$ R. J. Schutz, in VLSI Technology, 2nd ed., edited by S. M. Sze (McGrawHill, New York, 1988), p. 184.

${ }^{2}$ E. Kay, J. W. Coburn, and A. Dilks, in Topics in Current Chemistry, edited by S. Veprek and M. Venugopala (Springer, New York, 1980), p. 1.

${ }^{3}$ R. A. Barker, T. M. Mayer, and W. C. Pearson, J. Vac. Sci. Technol. B 1, 37 (1983).

${ }^{4}$ D. C. Gray, I. Tepermeister, and H. H. Sawin, J. Vac. Sci. Technol. B 11, 1243 (1993).

${ }^{5}$ T. M. Mayer and R. A. Barker, J. Vac. Sci. Technol. 21, 757 (1982).

${ }^{6}$ R. A. Gottscho, C. W. Jurgensen, and D. J. Vitkavage, J. Vac. Sci. Technol. B 10, 2133 (1992).

${ }^{7}$ E. Zawaideh and N. S. Kim, J. Appl. Phys. 62, 2498 (1987).

${ }^{8} \mathrm{P}$. Hoffmann and F. Heinrich, in Proceedings of the 9th International Symposium on Plasma Chemistry (Pugnochiuo, Italy, 1989), p. 1003.

${ }^{9}$ F. Fracassi, E. Occhielo, and J. W. Coburn, J. Appl. Phys. 62, 3980 (1987).

${ }^{10}$ J. Ding, J.-S. Jenq, G.-H. Kim, H. L. Maynard, J. S. Hamers, N. Hershkowitz, and J. W. Taylor, J. Vac. Sci. Technol. A 11, 1283 (1993).

${ }^{11}$ N. Mutsukura, S.-I. Inoue, and Y. Machi, J. Appl. Phys. 72, 43 (1992).

${ }^{12}$ W. D. Partlow, R. E. Witkowski, R. M. Young, R. Fedder, and F. Hagedron, SPIE Proc. 1325, 258 (1990).

${ }^{13}$ J. W. Zou, K. Reichdelt, K. Schmidt, and B. Dischlet, J. Appl. Phys. 65, 3914 (1989).

${ }^{14}$ J. W. Zou, K. Schmidt, K. Reichdelt, and B. Dischlet, J. Appl. Phys. 67, 487 (1990).

${ }^{15}$ J.-S. Jenq, J. Ding, J. W. Taylor, and N. Hershkowitz, Plasma Sources Sci. Technol. 3, 154 (1994).

${ }^{16}$ R. L. McClain, Ph.D. thesis, University of Wisconsin-Madison, 1991 (unpublished). 